\title{
Control of Genetically Prescribed Protein Tyrosine Kinase Activities by Environment-Linked Redox Reactions
}

\author{
Izumi Nakashima, Yoshiyuki Kawamoto, Kozue Takeda, and Masashi Kato \\ Department of Biomedical Sciences, College of Life and Health Sciences, Chubu University, Kasugai, Aichi 487-8501, Japan \\ Correspondence should be addressed to Izumi Nakashima, inakashi@isc.chubu.ac.jp
}

Received 25 January 2011; Revised 18 April 2011; Accepted 28 April 2011

Academic Editor: Robert Z. Qi

Copyright ( $) 2011$ Izumi Nakashima et al. This is an open access article distributed under the Creative Commons Attribution License, which permits unrestricted use, distribution, and reproduction in any medium, provided the original work is properly cited.

\begin{abstract}
Recent observations on environment-linked control of genetically prescribed signaling systems for either cell activation or cell death have been reviewed with a focus on the regulation of activities of protein tyrosine kinases (PTKs). The environmentlinked redox reactions seem to primarily affect cell surface receptors and cell membrane lipid rafts, and they induce generation of reactive oxygen species (ROS) in cells. ROS thus generated might upregulate the catalytic activities of PTKs through inactivating protein tyrosine phosphatases that dephosphorylate and inactivate autophosphorylated PTKs. Recent evidence has, however, demonstrated that ROS could also directly oxidize SH groups of genetically conserved specific cysteines on PTKs, sometimes producing disulfide-bonded dimers of PTK proteins, either for upregulation or downregulation of their catalytic activities. The basic role of the redox reaction/covalent bond-mediated modification of protein tertiary structure-linked noncovalent bondoriented signaling systems in living organisms is discussed.
\end{abstract}

\section{Introduction}

Enzymes, of which structures and functions are prescribed in genes, basically mediate all metabolisms needed for the development and functions of living organisms. Among the many enzymes, protein tyrosine kinases (PTKs) are known to play key roles in intracellular signaling for the development and functions of cells [1]. The mechanisms for regulation of the catalytic activities of PTKs seem to be principally prescribed in genes. These mechanisms include phosphorylation and dephosphorylation of specific tyrosine residues on the kinase protein by other PTKs or protein tyrosine phosphatases (PTPs), the structures and functions of which are all under genetic control [2-8]. For example, the catalytic activity of Src, a representative PTK, is known to be downregulated through phosphorylation of Tyr527 located in the tail of Src protein by CSK, another PTK, which leads to binding of the tail to the specific structure on the SH2 domain of Src to make the catalytic domain of Src closed [2, 3]. Additionally, it is known to be upregulated by dephosphorylation of phosphorylated Tyr527, which allows the catalytic domain to open, inducing phosphorylation of Tyr416 as the major autophosphorylation site and upregulation of the kinetic activity to phosphorylate exogenous specific substrates $[2-4,7,8]$. On the other hand, dephosphorylation of phosphorylated Tyr416 by PTPs downregulates the kinase activity $[5,6,8]$.

Results of several early studies have, however, suggested that the catalytic activities of PTKs are modulated by exposure of cells to environmental chemicals or oxidative agents [9-13]. It could be that the catalytic activities of PTKs are upregulated through redox mechanism-based inactivation of PTPs which otherwise dephosphorylate phosphorylated tyrosines at the major autophosphorylation sites of PTKs [11-14]. Recent evidence has, however, also suggested that environment-linked chemical or redox reactions directly attack PTKs for modification of their catalytic activities and subsequent signaling for cell activation [15-19]. This may occur through oxidization of $\mathrm{SH}$ groups of specific cysteines on PTK molecules by ROS that are generated intracellularly after clustering of cell surface receptors in association with membrane lipid rafts by environment-linked chemical or 
redox reactions $[15,20-24]$. Chemical or redox reactionmediated clustering of cell surface receptors in association with membrane lipid rafts has further been shown to deliver signals for either cell activation or cell death depending on the conditions [20, 24-30].

Recent observations on how environment-linked chemical or redox reactions control genetically prescribed signaling for cell activation or cell death are reviewed here, following several related review articles on this matter $[9,10,28$, 31-38]. Additionally, significance of these observations is briefly discussed from the viewpoint of crosstalk between environment-linked chemical or redox reaction- (covalent bond)-mediated and genetically prescribed (protein tertiary structure-linked noncovalent bond-oriented) regulation of intracellular signaling for either cell activation or cell death.

\section{Environment-Linked Chemical or Redox Reaction-Mediated Bypass of Genetically Prescribed Receptor-Ligand Recognition}

Initial activation of PTKs is usually linked to recognition by cell surface receptors of specific ligands. Thus, receptor type PTKs such as EGFR first bind specific ligands such as EGF to be dimerized on cell membranes for activation. On the other hand, Src family PTKs as nonreceptor type PTKs with a hydrophobic structure at the N-terminus are activated following specific ligand-induced clustering of cell surface receptors in association with membrane lipid rafts. Lipid rafts are known to work as a station of molecules for signal transduction to which the nonreceptor type PTKs are attached under the cell membrane [39-43]. Earlier experiments showed that this receptor-ligand bondmediated step for activation of either receptor type [26] or Src family nonreceptor type [17, 20-23, 44, 45] PTKs in cells can be bypassed by environment-linked chemical or redox reactions. These reactions induce clustering of receptors and membrane lipid rafts, which somewhat mimics ligand-mediated receptor clustering. Environmental agents for such chemical or redox reactions include thiol-reactive $\mathrm{Hg}^{2+}$ ions [20-22, 44-46], arsenite [24, 29] and methane sulfonate compounds (1,4-butanediyl-bismethanethiosulfonate (BMTS) [45, 47], protein amino group-reactive carbonyl compounds (glyoxal, methylglyoxal) [23, 48, 49], and protein sulfhydryl, amino, and imidazole groups-reactive 4hydroxynonenal (HNE) [26, 27, 50].

This pathway of activation of PTKs has been most extensively studied in cells carrying Src family PTKs such as c-Src (primary and tertiary structures of human c-SRC are illustrated in comparison with those of human c-RET in Figure 1) and Lck, which direct other PTKs such as ZAP70 and Syk and a large family of protein serine/threonine kinases downstream of the signal transduction cascade. Exposure of cells carrying c-Src or Lck to $\mathrm{Hg}^{2+}$, arsenite, glyoxal, or 1,4-butanediyl-bismethanethiosulfonate (BMTS) induced chemical or redox reaction-mediated clustering of membrane rafts. This occurred in close association with clustering of glycosylphosphatidyl inositol- (GPI-) anchored proteins in the rafts as the potential direct targets of the redox reactions and Src family PTKs that are bound to lipid rafts with a myristoylated membrane-targeting structure at the $\mathrm{N}$ terminus $(20,53)$. This signaling event was followed by activation of Src family PTKs as well as generation of ROS [23, 46]. Generation of ROS possibly underlay the mechanism of activation of PTKs and ultimately led to cell activation or cell death. All of these signal transduction pathways were inhibited by methyl beta cyclodextrin, a compound that disrupts lipid rafts by removing cholesterol from membranes [24]. This suggested a crucial requirement of the integrity of membrane lipid rafts in the redox reaction-linked signal pathway that apparently mimics the genetically prescribed receptor-ligand bond-mediated signal transduction. HNE, which is able to pass through the plasma membrane, also triggered another signal transduction pathway for cell death, possibly through scavenging intracellular glutathione [27]. These results suggest that genetically prescribed signal transduction pathways for cell activation or cell death, including the step of PTK activation, can be bypassed or modified at different steps by environment-linked chemical or redox reaction-mediated pathways.

As briefly mentioned in the introduction section, the receptor-ligand bond-mediated signaling pathway for activation of Src kinase is originally prescribed by genes. This pathway includes phosphorylation of Tyr527 in the tail of Src protein by CSK to be bound to the SH2 domain of Src for stabilizing and inactivating the kinase molecule [2$4,7]$. Binding between cell surface receptors attached to membrane lipid rafts, including GPI-anchored proteins, and their ligands may trigger the signal pathway for dephosphorylation of phosphorylated Tyr527 of Src kinase (or its equivalent tyrosine residues of other Src family PTKs) by specific PTPs. This should result in disruption of the bond between phosphorylated Tyr527 and SH2 domain of Src and induction of autophosphorylation of Tyr416 for activation of the catalytic activities of Src or other Src family PTKs $[5,6,8]$. Recently, the question of whether this is the only mechanism for triggering Tyr416 autophosphorylation and activation of Src or other Src family PTKs has been repetitiously discussed [10, 31-37].

$\mathrm{Pu}$ et al. [44] investigated whether the environmentlinked chemical or redox reaction-oriented signaling pathway that bypasses the genetically prescribed cell surface receptor-ligand interaction-mediated pathway includes the above-described Tyr527-mediated regulatory mechanism of Src kinase. They revealed that cells carrying Tyr527-missing $\mathrm{v}$-Src or cells missing CSK for phosphorylation of Tyr527, which are thereby already active, were still reactive to $\mathrm{Hg}^{2+}$ for superactivation of the kinases. It was concluded from these results that the environment-linked chemical or redox reaction-mediated pathway triggers a new mechanism for Tyr416 autophosphorylation and activation of Src kinase independent of dephosphorylation of previously phosphorylated Tyr 527.

Oxidative agents are known to inactivate PTPs of which cysteine residues in the catalytic domain [51] are sensitive to the agents. It has long been suggested that this is the central or even the only mechanism of upregulation of PTKs by oxidative agents, maintaining the autophosphorylation level 
of major tyrosine residues in the catalytic domain of PTKs $[11,12,52]$. ROS produced in cells through the mechanism triggered by environment-linked chemical or redox reactionmediated clustering of cell surface receptors/membrane rafts might actually inactivate PTPs, which in turn upregulate the catalytic activity of PTKs [14].

\section{Redox Reaction-Mediated Direct Modification of Molecular Structures of PTKs for Regulation of Their Catalytic Activities In Vitro}

In earlier experiments, $\mathrm{Pu}$ et al. [15] and Akhand et al. [16] exposed Src kinase proteins that had been immunoprecipitated from cell lysates in a medium with detergents to sulfhydryl-reactive $\mathrm{Hg}^{2+}$ [53] or nitric oxide- (NO-) generating S-nitroso-N-acetyl penicillamine (SNAP) [16]. They then measured the catalytic activities of Src proteins for Tyr416 autophosphorylation and tyrosine phosphorylation of specific substrates.

$\mathrm{Pu}$ et al. [15] showed that the catalytic activity of immunoprecipitated c-Src from NIH3T3 cells was elevated severalfold by exposure to low to moderate concentrations $(0.5-50 \mu \mathrm{M})$ of $\mathrm{Hg}^{2+}$, whereas it was decreased by exposure to a high concentration $(500 \mu \mathrm{M})$ of $\mathrm{Hg}^{2+}$. Nacetylcysteine neutralized this $\mathrm{Hg}^{2+}$ effect, suggesting the involvement of a redox reaction in the mechanism. Addition of the protein tyrosine phosphatase inhibitor $\mathrm{Na}_{3} \mathrm{VO}_{4}$ to the reaction mixture did not inhibit the $\mathrm{Hg}^{2+}$-mediated activation. This result provisionally eliminates the possibility that $\mathrm{Hg}^{2+}$-mediated inactivation of PTPs was the central mechanism for upregulation of the c-Src activity. $\mathrm{Hg}^{2+}$ was further found to be capable of activating Tyr527-defective $\mathrm{v}$-Src kinase and c-Src kinase from mutant cells defective in Tyr527-phosphorylating Csk kinase. Cyanogen bromide cleavage maps revealed that $\mathrm{Hg}^{2+}$ selectively promoted the autophosphorylation at Tyr416 and that the previously in vivo radiolabeled phosphorus on Tyr527 was not deleted. These observations suggested that $\mathrm{Hg}^{2+}$ triggered the redox reaction-linked mechanism for promotion of both autophosphorylation and general catalytic activity of Src kinase in vitro independent of Tyr527-linked or PTP-mediated regulation. The exact target structure of the redox reaction for promotion of autophosphorylation of c-Src was not, however, identified in these experiments.

Exposure of Src kinase to SNAP was also shown to promote Tyr416 autophosphorylation independent of Tyr527linked or PTP-mediated regulation [16]. Interestingly, promotion of Tyr416 autophosphorylation, which accompanied increase in overall kinase activity of c-Src, was found to be closely linked to S-S bond-mediated aggregation of Src molecules. This observation supported a hypothetical view that the environment-linked oxidative agent-triggered signal transduction pathway for upregulation of c-Src activity includes sulfhydryl-oxidation-mediated structural modification of Src proteins.

Further experiments demonstrated that exposure of cells carrying RET kinases to ultraviolet (UV) $[17,54]$ or osmotic pressure [18] intracellularly induced activation of these kinases. Either c-RET proto-oncogene as a receptor type PTK or its consistently active oncogene mutants, including an extracellular domain-deleted (nonreceptor type) mutant RET-PTC-1 (primary and tertiary structures of c-RET in comparison with those of c-SRC are illustrated in Figure 1), were activated. Interestingly, UV- or osmotic pressuremediated activation of RET-PTC-1 accompanied an increase in the amount of S-S bond-mediated dimers of the kinase proteins. Exposure of cells to UV or osmotic stress increased $\mathrm{S}-\mathrm{S}$ bond-mediated dimerization from $1 \%$ as the background level to $4 \%$ of total RET-PTC-1 proteins, and the fraction of dimerized RET-PTC-1 selectively displayed a high level of autophosphorylation $[17,18]$. This was demonstrated by an in vitro kinase assay under the condition with or without a reducing agent (2-mercaptoethanol) for the kinase proteins that had been immunoprecipitated from the UV- or osmotic stress-exposed cells. It was speculated that the UVinduced or osmotic-stress-generated ROS in cells attacked intracellular domains of the RET kinase to oxidize the domains for S-S bonded dimerization, ultimately resulting in promotion of autophosphorylation and activation of the kinase.

More recently, direct evidence has been provided by Kemble and Sun [19,38] for oxidative modification of purified Src proteins accompanying changes in the levels of their catalytic activities. They isolated Src proteins from bacteria lysates containing recombinant Src proteins by adding iminodiacetic acid-agarose beads charged with $\mathrm{NiCl}_{2}$ to the lysates. The isolated Src proteins were active when reduced by adding dithiothreitol (DTT) to a medium containing Src proteins and retained $8-25 \%$ of their full activity when oxidized by adding $\mathrm{H}_{2} \mathrm{O}_{2}$ to the medium. This downregulation of the kinase activity was shown to be caused by oxidation of specific cysteine residues on Src, resulting in an S-S bridge for dimerization of Src molecules. Their study results formally proved that some cysteine residues on Src proteins are capable of being oxidized for dimer formation with downregulation of the kinase activity. This effect of dimer formation is, however, apparently opposite to the above-described effect on immunoprecipitated RETPTC-1 kinase, in which the kinase activity was upregulated in linkage to $\mathrm{S}-\mathrm{S}$ bond-mediated dimerization of kinase proteins $[17,18]$.

The reason why dimer formation of Src or RET-PTC-1 due to $\mathrm{S}$-S bonds between specific cysteine residues induced apparently opposite effects on their kinase activities in the experiments performed by two different study groups [1719] is not clear. However, in the model of RET-PTC-1, kinase molecules must have been exposed to possibly mildly oxidative environments in cells. Intracellular environments are normally maintained for reducing because of the presence of large amounts of reducing agents such as glutathione and thioredoxin, and the oxidizing/reducing balance in cells therefore does not change drastically even after production of ROS in cells (57). The mildly oxidative environment possibly selectively affected $\mathrm{SH}$ groups of specific cysteines for fine structural modification of kinase proteins, which was needed for maintenance and upregulation of the kinase activity. 

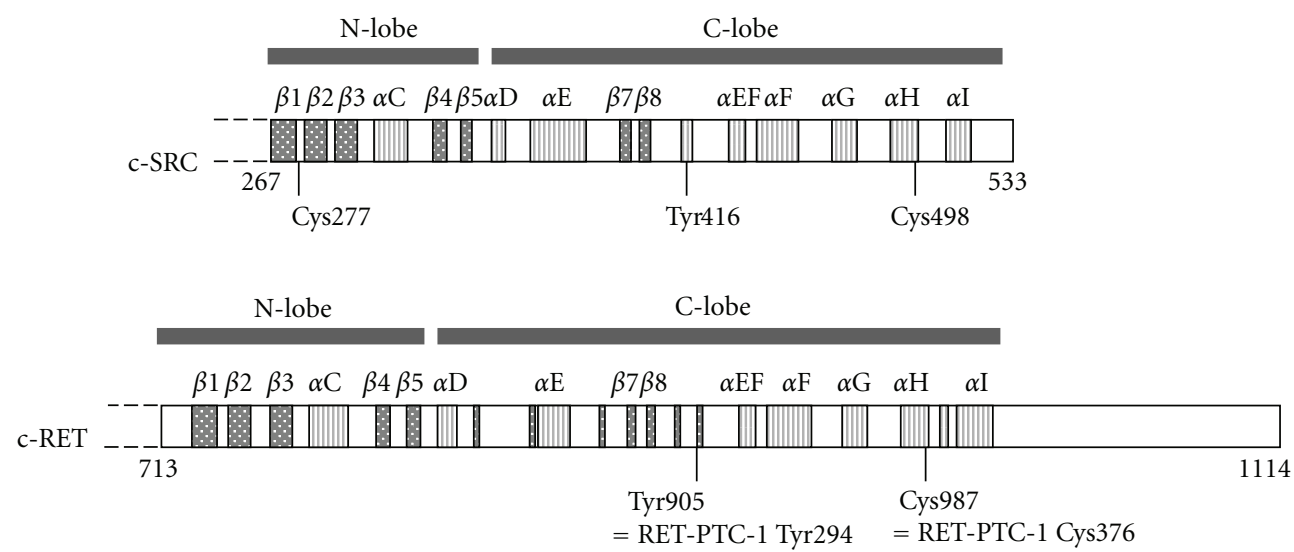

(a)
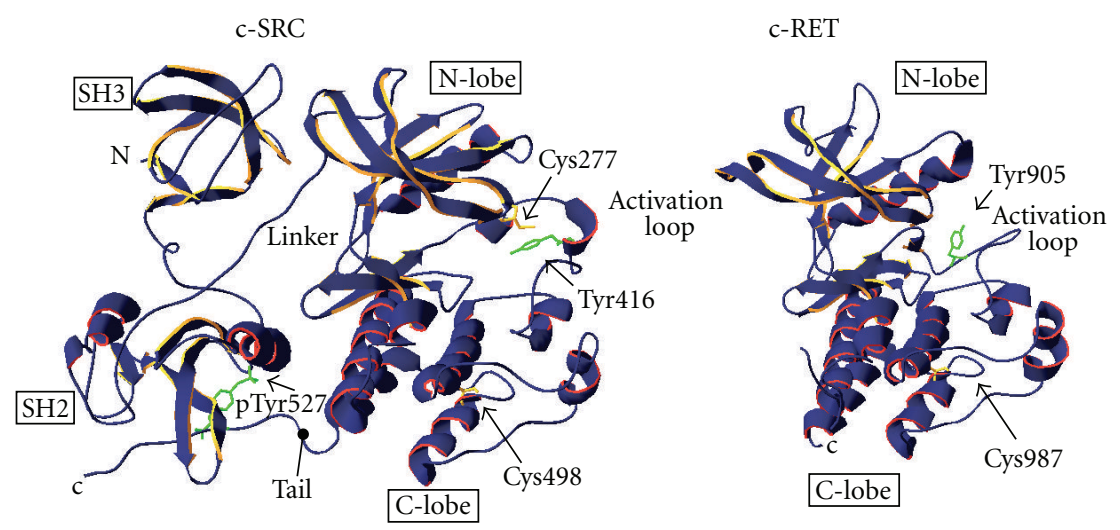

(b)

FIGURE 1: Shematic illustration of structures of human c-SRC and human c-RET kinase proteins. Primary and tertiary structures of c-SRC (PDB 2SRC) and c-RET (PDB 2IVS) are deduced from the data in Protein Data Bank (PDB). (a) Primary structures of c-SRC (top) and c-RET (bottom) kinase domains. $\alpha$ : $\alpha$-helix; $\beta$ : $\beta$-sheet. (b) Tertiary structures of SH3, SH2, and kinase (N-lobe and C-lobe) domains of c-SRC (left) and kinase domain of c-RET (right). Positions of Tyr416 of c-SRC and Tyr905 of c-RET as major autophosphorylation sites and those of Cys277/Cys498 of c-SRC and Cys987 of c-RET (equivalent to Cys376 of RET-PTC-1) as well as positions of N-terminal (N) and C-terminal (C) amino acids are shown.

RET-PTC-1 molecules were possibly located in a row under the cell membrane, which should make two neighboring kinase molecules easily accessible to each other for S$S$ bonded dimerization at specific cysteines. S-S bonded dimerization in close linkage to upregulation of the kinase activity $[17,18]$ is direct evidence of the involvement of oxidative structural modification in redox-mediated upregulation of PTK activities. S-S bonded dimerization may not, however, be an absolute requirement for the oxidative effects for upregulation of PTKs as discussed in the next section.

In contrast, isolated recombinant Src proteins prepared by Kemble and Sun [19], which were probably arranged in a random manner in vitro, were affected by rather heavily oxidative agents such as oxygen in air and $\mathrm{H}_{2} \mathrm{O}_{2}$, which possibly oxidized free $\mathrm{SH}$ groups on multiple cysteines on Src but selectively induced dimerization through S-S bonds between specific cysteines, probably inducing a major structural change of Src for downregulation of the kinase activity.

\section{Identification of Submolecular Structures in PTK Molecules as the Targets of Redox Reaction for Regulating the Catalytic Activities of PTKs}

Kemble and Sun [19] studied the target cysteine residues responsible for S-S bonded dimer formation of Src proteins by preparing mutant proteins in which each of totally ten cysteine residues of Src was replaced with alanine. They showed that dimer formation occurred only through oxidation of Cys277, which is located in the catalytic domain of Src.

On the other hand, Kato et al. $[17,54]$ and Takeda et al. [18] examined the sensitivity to ultraviolet light 
irradiation or osmotic stress of RET-PTC-1 (a nonreceptor type PKC) mutants. Each of two cysteine residues (Cys365 and Cys376) on the alpha-helix $\mathrm{H}$ region downstream of the catalytic domain of RET-PTC-1 was replaced with alanine. They found that the mutants in which Cys376 of RETPTC-1 is replaced with alanine almost totally lost their background ability for inducing autophosphorylation of Tyr294 as the major autophosphorylation site [55]. The mutants at the same time lost their abilities to form S-S bonded dimers of kinase proteins in cells to which a high level of autophosphorylation was associated (in vivo), even after exposure to ultraviolet irradiation or osmotic stress. These results are in contrast to another observation that replacement of Cys365 of RET-PTC-1, a cysteine located near Cys 376 on alpha-helix $\mathrm{H}$, with alanine did not detectably alter either background ability for autophosphorylation of Tyr294 or the ability to form S-S bonded dimer formation in cells. Correspondingly, a single mutation in Cys498 of v-Src, an equivalent of Cys376 of RET-PTC-1, but not in other cysteines yielded clear suppression of kinase activity and temperature sensitivity in cell transformation [56]. Takeda et al. [57] further confirmed that all RET-PTC-1 mutants in which C376 of RET-PTC-1 was replaced with glycine, lysine, threonine or serine lost their background level of activity for autophosphorylation of Tyr294 in cells, even though comparable amounts of RET proteins were obtained from each of these mutants, suggesting that possibly accelerated degradation of the mutant proteins is not the reason for the above-mentioned loss of abilities. All of these results suggested that Cys376 of RET-PTC-1, an equivalent of Cys498 of Src or Cys987 of c-RET, is crucially involved in the maintenance and upregulation of catalytic activities of these kinase proteins in cells. Moreover, because both SS bonded dimer formation and Tyr294 autophosphorylation were simultaneously lost in the Cys376-missing RET-PTC1 mutant, it is likely that Cys 376 plays a crucial role in the redox regulation of the kinase activity of this PTK through oxidative structural modification.

Cys277 of Src is located in the sequence context of GQGCFG on the small N-terminal lobe of the catalytic domain and is conserved in 8 of more than 90 human PTKs examined [19]. On the other hand, Cys376 of RETPTC-1 or Cys498 of Src is located in the MXXCW motif on the C-terminus of alpha-helix $\mathrm{H}$ and is conserved in all but one (FGR: threonine in place of cysteine) of 82 human PTKs examined in the database Swiss-Prot (18/11/2004), suggesting a crucial role in regulation of the kinase activity of PTKs in general $[32,33,55]$.

Structural modification of Cys376 of RET-PTC-1, Cys498 of Src or its equivalent cysteine residues on almost all PTKs in a mildly oxidative environment in the cell could thus work to basically maintain or to upregulate the kinase activity. In contrast, modification of Src proteins at Cys277 or its equivalent cysteine residues on 8 out of more than 90 human PTKs in possibly heavily oxidative environments evidently acted to downregulate it (see Figure 2). The reason of the different effects of S-S linked dimerization on RET-PTC1 and Src might simply be that RET-PTC-1 is missing the cysteine equivalent to Cys 277 of Src. Pu et al. [15] have, however, shown that exposure of immunoprecipitated Src proteins to low to moderate levels of sulfhydryl-reactive $\mathrm{Hg}^{2+}$ in vitro caused upregulation of the kinase activity, whereas exposure of these proteins to a high level of $\mathrm{Hg}^{2+}$ downregulated the activity. The latter observation might have involved oxidization of Cys277, although S-S bonded dimerization was not detected in their experiment. It is possible that long-term exposure to oxygen in air (in vitro) during preparation of recombinant Src proteins [19] caused the proteins to be oxidized at a number of free SH groups of cysteines possibly including both Cys277 and Cys498. S-S bonded dimerization, however, occurred only between Cys277, of which the downregulatory effect mostly overcame the potential upregulatory effect by oxidization of Cys498.

Taken together, the results indicate that oxidative structural modification of PKTs might bidirectionally control their kinase activities in the following manner. Fine structural modification by oxidization of Cys376 of RET-PTC-1 or its equivalent cysteines in mildly oxidative environments works for maintenance and upregulation of kinase activities of almost all PTKs carrying equivalent cysteine residues. In contrast, more extensive structural modification of PTKs through oxidization of Cys 277 of Src or its functionally equivalent cysteine residues on other PTKs in heavily oxidative environments downregulates their kinase activities. The fine structural modification through oxidization of Cys376 of RET-PTC-1, Cys498 of Src or its equivalent cysteine residues on other PTKs for kinase activation may not necessarily require $\mathrm{S}-\mathrm{S}$ bonded dimerization of kinase proteins. Oxidization of the SH group to $\mathrm{S}-\mathrm{X}$ seems still effective, because exposure of immunoprecipitated Src proteins to sulfhydrylreactive $\mathrm{Hg}^{2+}$ successfully induced upregulation of the kinase activity without S-S bonded dimerization of the kinase proteins in vitro.

\section{Further Crosstalk between Genetically Prescribed and Environment-Linked Signaling Systems for Regulating Kinase Activities of PTKs}

FGR, an exceptional PTK that does not carry Cys376 of RETPTC-1-equivalent, carries threonine in place of cysteine in the MXXCW motif. Takeda et al. [57] therefore investigated the mechanism for this exceptional PTK to keep its kinase activity. They prepared a number of RET-PTC-1 mutants in which Cys376 was replaced with glycine, lysine, threonine, or serine and examined the kinase activities of imunoprecipitated mutant RET-PTC-1 from cell lysates in vitro. As described in the previous section, all of these mutant proteins almost totally lost their basic kinase activities in vitro. Surprisingly, however, cells carrying Cys376Ser or Cys376Thr mutant RET-PTC-1, but not those carrying Cys376Gly or Cys376Lys mutant RET-PTC-1, displayed almost normal levels of autophosphorylation at Tyr294 in vivo (in cells). Correspondingly, Cys376Thr/Ser, but not Cys376Gly/Lys, was capable of inducing cell transformation almost as effectively as parental RET-PTC-1 as an active oncogene. 


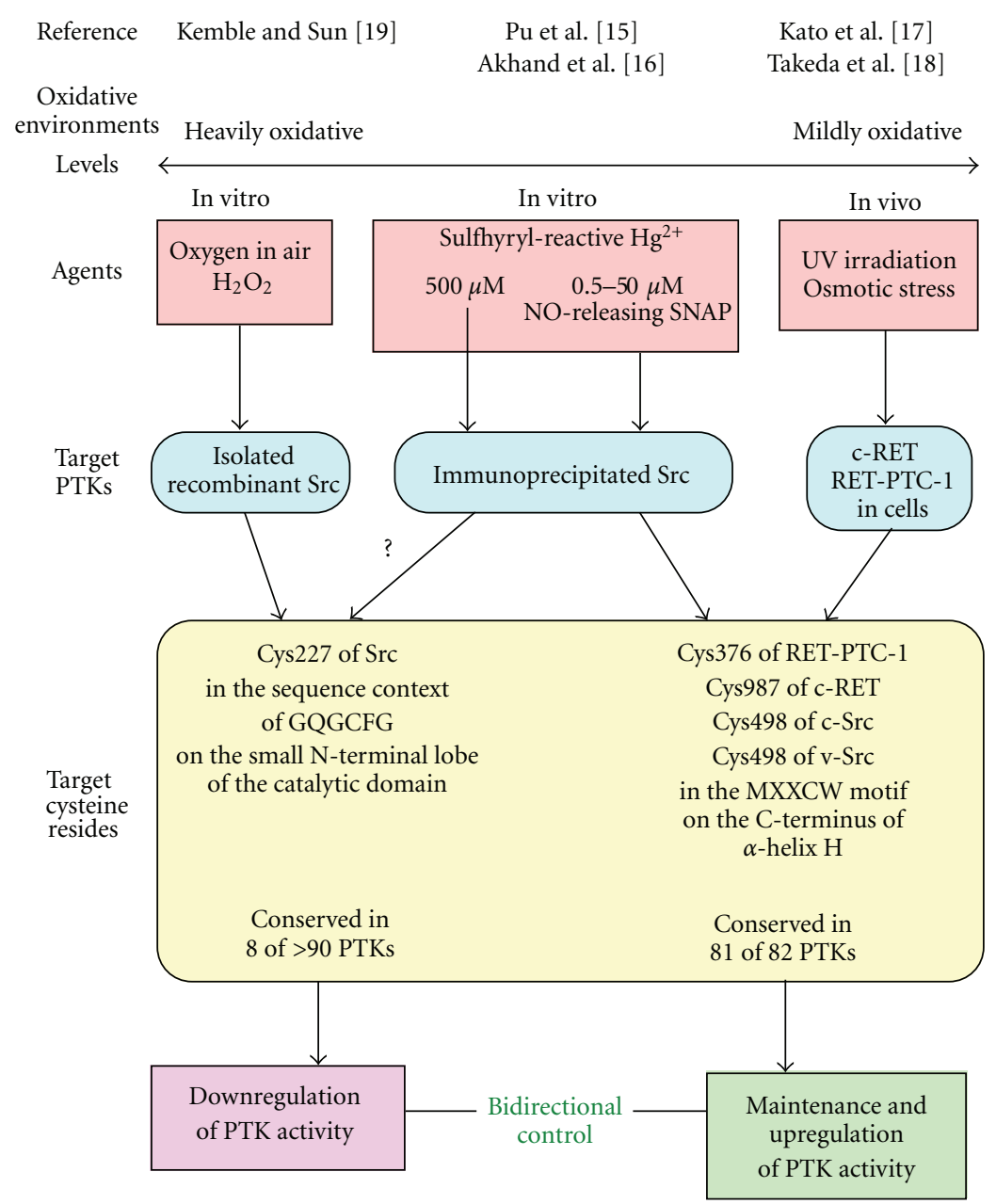

FIgURE 2: Summary of observations for bi-directional control of PTKs through their potential structural modification by redox reactions. Kemble and Sun demonstrated a pathway for definitive structural modification-mediated downregulation of the kinase activity of Src in heavily oxidative environments in vitro through disulfide-bonded dimerization of Src proteins at Cys277. On the other hand, Kato et al. and Takeda et al. revealed another pathway for possibly fine structural modification-mediated upregulation of kinase activities of RET-PTC-1 in a mildly oxidative environment in vivo through disulfide-bonded dimerization of RET proteins at Cys376. Pu et al. and Akhand et al. further demonstrated that exposure of immunoprecipitated Src proteins from cell lysates to low to moderate levels of sulfhydryl-reactive $\mathrm{Hg}^{2+}$ or NO-releasing SNAP induced Tyr527/PTP-independent activation of Src kinase, whereas their exposure to a high level of $\mathrm{Hg}^{2+}$ downregulated it.

Further study demonstrated that the essential role of the cysteine at the MXXCW motif in initiating the kinase activity of RET-PTC-1 could be partially replaced by the activity of protein kinase $\mathrm{C}$ as a serine/threonine kinase in vivo (but not in vitro). This result suggested that the PTK missing the cysteine at the MXXCW motif uses an alternate PKC-mediated pathway as a rescue system for maintaining the kinase activity. PKC itself in the rescue pathway should, however, be activated by another PTK that possesses the cysteine at the MXXCW motif. It is therefore speculated that there is a new pathway of crosstalk between genetically prescribed and environment-linked mechanisms for regulation of PTKs. Crosstalk between Src and RET kinases has also been reported as Src-mediated repair of function-impaired RET-MEN2A (another oncogenic RET mutant) with substitutions of tyrosines in the $\mathrm{COOH}$ terminal kinase domain for phenylalanine [58].

\section{Crosstalk between Environment-Linked and Genetically Prescribed Signals for Either Cell Activation Or Cell Death}

Environment-linked oxidative signals have also been shown to induce apoptotic cell death, basically sharing a common initial step for cell activation, by inducing clustering of cell surface receptors in association with membrane lipid rafts [24]. This signaling pathway was shown to induce generation of reactive oxygen species (ROS) and activation of apoptosis signal-regulating kinase 1 (ASK-1), c-Jun amino-terminal 


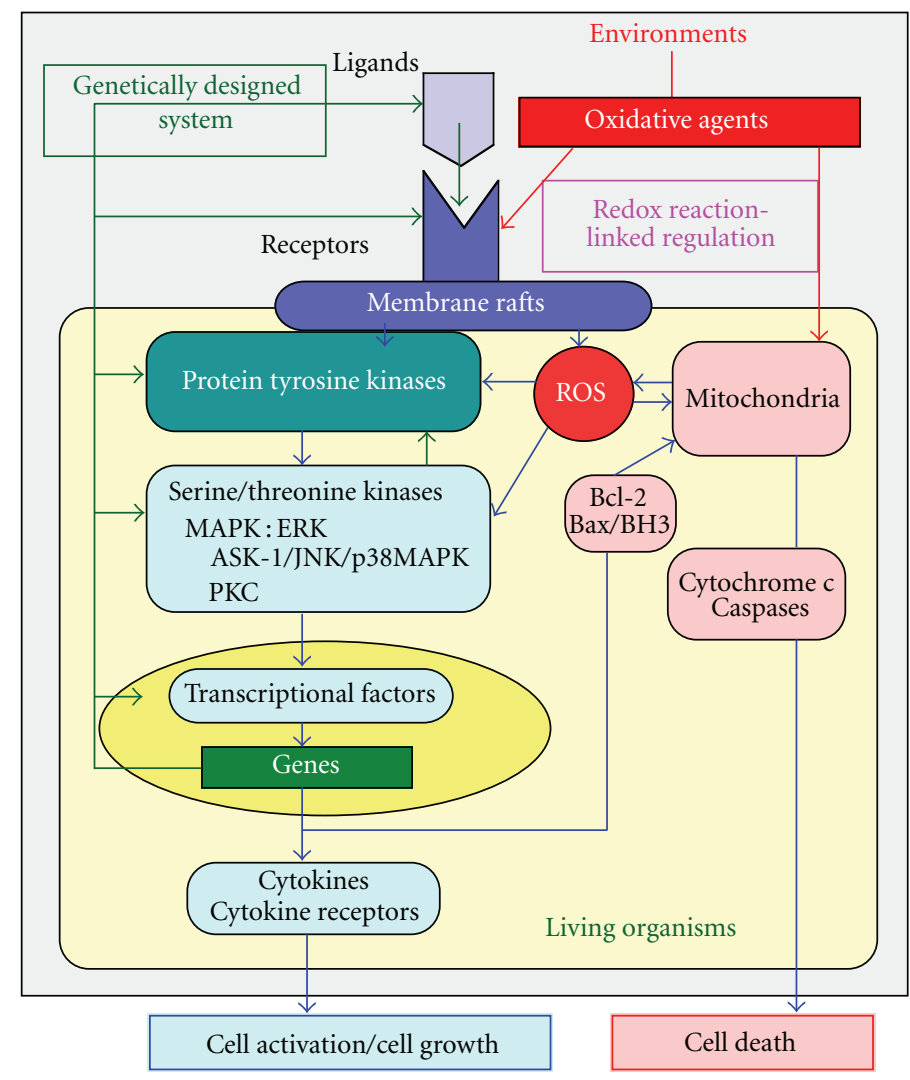

FIGURE 3: Suggested crosstalk between the genetically prescribed system in living organisms and environments through chemical or redox reactions in the signal transduction for either cell activation or cell death. The genetically prescribed signaling system includes noncovalent bond-oriented interaction between receptors and specific ligands and subsequent activation of PTKs, serine/threonine kinases, and transcriptional factors. This system is regulated by environment-linked covalent bond-mediated redox reactions, which primarily attack cell surface receptors in association with cell membrane lipid rafts, resulting in intracellular production of ROS. ROS might directly or indirectly affect PTKs, ASK-1, or mitochondria for promotion of subsequent signal transduction, which ultimately leads to either cell activation/growth or cell death.

kinase (JNK), and p38 MAPK and reciprocal regulation of Bcl-2/Bax $[24,48,50,59,60]$ or downregulation of ERK [49]. This pathway was further shown to cause mitochondria to release cytochrome $\mathrm{c}$, leading to activation of caspases for DNA fragmentation and apoptotic cell death, or to release of a high level of ROS for necrotic cell death [25]. Environmentlinked oxidative agents might also more directly affect mitochondria [30] or induce an intracellular reduced glutathione level [27], ultimately leading to apoptotic or necrotic cell death.

These observations suggest extensive crosstalk between microenvironment-linked and genetically prescribed signals for inducing either cell activation or cell death (see Figure 3). Switching for either cell activation or cell death seems to depend on the nature and levels of environment-linked chemical or oxidative signals and on the time of involvement of environment-linked signals in relation to the stage of genetically prescribed development and functions of the cells.

A number of environment-linked chemical or oxidative signals seem to be changed to intracellular oxidative signals through generation of ROS as potential second messengers in signal transduction in cells $[34,35]$. ROS may lead to either cell activation or cell death depending largely on the level of ROS generated $[20,25]$. Thus, a low level of environment-linked oxidative stress probably induces a low grade of ROS production for upregulation of PTKs. This causes downstream activation of MAP family kinases such as ERK, serine/threonine kinases, and transcriptional factors for production of specific cytokines and cytokine receptors, ultimately leading to cell activation and proliferation. In contrast, a slightly increased level of oxidative stress induces apoptotic cell death through a cascade of signaling such as a higher grade of ROS production and downstream activation of the ASK-1-mediated signal pathway including JNK/p35MAPK, Bax/BH3, cytochrome c, and caspases. Higher levels of stress induce necrosis accompanying a highgrade ROS production possibly through serious damage of mitochondria [47].

A low level of ROS is always produced by mitochondria and may be used for controlling the activation levels of PTKs physiologically [32]. A higher level of ROS can be produced in cells after direct attack of mitochondria by environmentlinked oxidative agents. Evidence has been provided for a 
mechanism to localize ROS signal at a specific subcellular compartment, which is essential for promoting redox-linked signaling pathways after cell surface receptor activation [61]. ROS production for inducing either cell activation or cell death has been suggested to be membrane raft linked [24, 62, 63 ] and might be localized through interactions of NADPH oxidase with signaling platforms associated with lipid rafts [61]. Favoring in part this speculation, Kawamoto et al. recently obtained experimental results suggesting that cells exposed to sulfhydryl-reactive arsenite generate ROS outside mitochondria (unpublished observation).

\section{A Potentially Basic Role of Environment-Linked Control of Genetically Prescribed Signaling Systems in Living Organisms}

Both the development and functions of living organisms are basically self-regulated by information natively included in genes. Thus, genes code for tertiary structures of proteins, including receptors and their ligands, which provide noncovalent bond-oriented molecular switches in signal transduction for either cell activation or cell death. However, the environment conditionally gives some covalent bondmediated nongenetical information to living organisms [9], particularly through environment-linked oxidizing or reducing (redox) reactions. The redox reactions are principally reversible and might therefore replace some of the genetically prescribed noncovalent bond-mediated molecular switches for signal transduction.

Recent evidence introduced here suggests that the environment-linked covalent bond (chemical or redox reaction-) mediated information plays a crucial role in controlling the genetically prescribed noncovalent bondoriented signaling systems for cell activation or cell death. The key target molecules for the covalent bond-mediated information are membrane lipid raft-associating cell surface receptors and intracellular PTKs. PTKs as crucial targets of redox regulation are capable of triggering subsequent cascade reactions of signaling elements such as protein serine/threonine kinases and transcription factors. The idea for general importance of redox regulation of PTKs in overall signal transduction in living organisms corresponds well to the fact that the specific cysteine residue as the target of redox regulation is highly conserved in a number of human PTKs [33].

\section{Conclusion}

Environment-linked chemical or redox reactions control the genetically prescribed signal delivery system for either cell activation or cell death in living organisms. Accumulated evidence indicates that this includes environment-mediated control of PTKs as a master key of intracellular signal transduction for cell activation and of several elements of signal transduction for cell death. The pathway for environment-linked control of PTKs include (1) clustering of cell surface receptors in association with membrane lipid rafts, potentially bypassing receptor-ligand interaction, and (2) membrane lipid raft-linked production of ROS beneath the membrane. This leads to (3) ROS-mediated inactivation of PTPs, which in turn upregulate PTKs, and to (4) ROS-mediated oxidative structural modification of PTKs for bi-directional direct control of PTKs through oxidization of $\mathrm{SH}$ groups of specific cysteines on PTKs to either S-S or S-X. Upregulated PTKs promote the subsequent signal transduction, ultimately resulting in either cell activation or cell death. Intracellular ROS produced might also promote other pathways of signal transduction for cell death. It is concluded that environment-linked chemical or redox reactions, which are mediated by covalent bonds, bidirectionally control the genetically prescribed and thereby protein tertiary structure-linked noncovalent bond-oriented signaling systems for either cell activation or cell death in living organisms.

\section{References}

[1] L. C. Cantley, K. R. Auger, C. Carpenter et al., "Oncogenes and signal transduction,” Cell, vol. 64, no. 2, pp. 281-302, 1991.

[2] A. Weiss, "T cell antigen receptor signal transduction: a tale of tails and cytoplasmic protein-tyrosine kinases," Cell, vol. 73, no. 2, pp. 209-212, 1993.

[3] J. A. Cooper and B. Howell, "The when and how of Src regulation," Cell, vol. 73, no. 6, pp. 1051-1054, 1993.

[4] W. Rodgers and J. K. Rose, "Exclusion of CD45 inhibits activity of p56 associated with glycolipid-enriched membrane domains," Journal of Cell Biology, vol. 135, no. 6 I, pp. 15151523, 1996.

[5] F. Sicheri, I. Moarefi, and J. Kuriyan, "Crystal structure of the Src family tyrosine kinase Hck," Nature, vol. 385, no. 6617, pp. 602-609, 1997.

[6] W. Xu, S. C. Harrison, and M. J. Eck, "Three-dimensional structure of the tyrosine kinase c-Src," Nature, vol. 385, no. 6617, pp. 595-602, 1997.

[7] M. L. Thomas and E. J. Brown, "Positive and negative regulation of Src-family membrane kinases by CD45," Immunology Today, vol. 20, no. 9, pp. 406-411, 1999.

[8] J. D. Ashwell and U. D’Oro, "CD45 and Src-family kinases: and now for something completely different," Immunology Today, vol. 20, no. 9, pp. 412-416, 1999.

[9] I. Nakashima, "Can cysteine direct tyrosine in signal transduction for environment-oriented gene control?" Nagoya Journal of Medical Science, vol. 59, no. 1-2, pp. 1-10, 1996.

[10] I. Nakashima, M. Y. Pu, A. A. Akhand, M. Kato, and H. Suzuki, "Chemical events in signal transduction," Immunology Today, vol. 18, no. 7, p. 362, 1997.

[11] H. Nakamura, K. Nakamura, and J. Yodoi, "Redox regulation of cellular activation," Annual Review of Immunology, vol. 15, pp. 351-369, 1997.

[12] P. Chiarugi and P. Cirri, "Redox regulation of protein tyrosine phosphatases during receptor tyrosine kinase signal transduction," Trends in Biochemical Sciences, vol. 28, no. 9, pp. 509$514,2003$.

[13] D. Barford, "The role of cysteine residues as redox-sensitive regulatory switches," Current Opinion in Structural Biology, vol. 14, no. 6, pp. 679-686, 2004.

[14] H. P. Monteiro, R. J. Arai, and L. R. Travassos, "Protein tyrosine phosphorylation and protein tyrosine nitration in 
redox signaling," Antioxidants and Redox Signaling, vol. 10, no. 5, pp. 843-889, 2008.

[15] M. Pu, A. A. Akhand, M. Kato et al., "Evidence of a novel redox-linked activation mechanism for the Src kinase which is independent of tyrosine 527-mediated regulation," Oncogene, vol. 13, no. 12, pp. 2615-2622, 1996.

[16] A. A. Akhand, M. Pu, T. Senga et al., "Nitric oxide controls Src kinase activity through a sulfhydryl group modificationmediated Tyr-527-independent and Tyr-416-linked mechanism," Journal of Biological Chemistry, vol. 274, no. 36, pp. 25821-25826, 1999.

[17] M. Kato, T. Iwashita, K. Takeda et al., "Ultraviolet light induces redox reaction-mediated dimerization and superactivation of oncogenic Ret tyrosine kinases," Molecular Biology of the Cell, vol. 11, no. 1, pp. 93-101, 2000.

[18] K. Takeda, M. Kato, J. Wu et al., "Osmotic stress-mediated activation of RET kinases involves intracellular disulfidebonded dimer formation," Antioxidants and Redox Signaling, vol. 3, no. 3, pp. 473-482, 2001.

[19] D. J. Kemble and G. Sun, "Direct and specific inactivation of protein tyrosine kinases in the Src and FGFR families by reversible cysteine oxidation," Proceedings of the National Academy of Sciences of the United States of America, vol. 106, no. 13, pp. 5070-5075, 2009.

[20] I. Nakashima, M. Y. Pu, A. Nishizaki et al., "Redox mechanism as alternative to ligand binding for receptor activation delivering disregulated cellular signals," Journal of Immunology, vol. 152, no. 3, pp. 1064-1071, 1994.

[21] M. Pu, L. Ma, K. Ohkusu et al., "Direct evidence of involvement of glycosylphosphatidylinositol-anchored proteins in the heavy metal-mediated signal delivery into T lymphocytes," FEBS Letters, vol. 361, no. 2-3, pp. 295-298, 1995.

[22] Y. Katano, M. Pu, A. A. Akhand et al., "Evidence of redoxlinked signaling for producing a giant signal complex," Journal of Cellular Biochemistry, vol. 57, no. 3, pp. 432-439, 1995.

[23] A. A. Akhand, M. Kato, H. Suzuki et al., "Carbonyl compounds cross-link cellular proteins and activate proteintyrosine kinase p60," Journal of Cellular Biochemistry, vol. 72, no. 1, pp. 1-7, 1999.

[24] K. Hossain, A. A. Akhand, M. Kato et al., "Arsenite induces apoptosis of murine $\mathrm{T}$ lymphocytes through membrane raft-linked signaling for activation of c-Jun amino-terminal kinase," Journal of Immunology, vol. 165, no. 8, pp. 4290-4297, 2000.

[25] A. A. Akhand, M. Kato, H. Suzuki, T. Miyata, and I. Nakashima, "Level of HgCI-mediated phosphorylation of intracellular proteins determines death of thymic Tlymphocytes with or without DNA fragmentation," Journal of Cellular Biochemistry, vol. 71, no. 2, pp. 243-253, 1998.

[26] W. Liu, A. A. Akhand, M. Kato et al., "4-hydroxynonenal triggers an epidermal growth factor receptor-linked signal pathway for growth inhibition," Journal of Cell Science, vol. 112, no. 14, pp. 2409-2417, 1999.

[27] W. Liu, M. Kato, A. A. Akhand et al., "4-hydroxynonenal induces a cellular redox status-related activation of the caspase cascade for apoptotic cell death," Journal of Cell Science, vol. 113, no. 4, pp. 635-641, 2000.

[28] A. A. Akhand, J. Du, W. Liu et al., "Redox-linked cell surfaceoriented signaling for T-cell death," Antioxidants and Redox Signaling, vol. 4, no. 3, pp. 445-454, 2002.

[29] K. Hossain, A. A. Akhand, Y. Kawamoto et al., "Caspase activation is accelerated by the inhibition of arsenite-induced, membrane rafts-dependent Akt activation," Free Radical Biology and Medicine, vol. 34, no. 5, pp. 598-606, 2003.
[30] K. Hossain, Y. Kawamoto, M. Hamada et al., "1,4-butanediylbismethanethiosulfonate (BMTS) induces apoptosis through reactive oxygen species-mediated mechanism," Journal of Cellular Biochemistry, vol. 108, no. 5, pp. 1059-1065, 2009.

[31] I. Nakashima, M. Kato, A. A. Akhand et al., "Redox-linked signal transduction pathways for protein tyrosine kinase activation," Antioxidants and Redox Signaling, vol. 4, no. 3, pp. 517-531, 2002.

[32] I. Nakashima, K. Takeda, Y. Kawamoto, Y. Okuno, M. Kato, and H. Suzuki, "Redox control of catalytic activities of membrane-associated protein tyrosine kinases," Archives of Biochemistry and Biophysics, vol. 434, no. 1, pp. 3-10, 2005.

[33] I. Nakashima, K. Takeda, Y. Kawamoto, Y. Okuno, M. Kato, and H. Suzuki, "MXXCW motief as a potential initiator of protein tyrosine kinases," in Trends in Protein Research, J. W. Robinson, Ed., pp. 119-131, Nova Science, Hauppauge, NY, USA, 2005.

[34] P. Chiarugi, "PTPs versus PTKs: the redox side of the coin," Free Radical Research, vol. 39, no. 4, pp. 353-364, 2005.

[35] J. V. Cross and D. J. Templeton, "Regulation of signal transduction through protein cysteine oxidation," Antioxidants and Redox Signaling, vol. 8, no. 9-10, pp. 1819-1827, 2006.

[36] P. Chiarugi and F. Buricchi, "Protein tyrosine phosphorylation and reversible oxidation: two cross-talking posttranslation modifications," Antioxidants and Redox Signaling, vol. 9, no. 1, pp. 1-24, 2007.

[37] P. Chiarugi, "Src redox regulation: there is more than meets the eye," Molecules and Cells, vol. 26, no. 4, pp. 329-337, 2008.

[38] G. Sun and D. J. Kemble, "To C or not to C: direct and indirect redox regulation of Src protein tyrosine kinase," Cell Cycle, vol. 8, no. 15, pp. 2353-2355, 2009.

[39] M. G. Low and A. R. Saltiel, "Structural and functional roles of glycosyl-phosphatidylinositol in membranes," Science, vol. 239, no. 4837, pp. 268-275, 1988.

[40] I. Stefanová, V. Horejsí, I. J. Ansotegui, W. Knapp, and H. Stockinger, "GPI-anchored cell-surface molecules complexed to protein tyrosine kinases," Science, vol. 254, no. 5034, pp. 1016-1019, 1991.

[41] I. Nakashima, Y. H. Zhang, S. M. J. Rahman et al., "Evidence of synergy between Thy-1 and CD3/TCR complex in signal delivery to murine thymocytes for cell death," Journal of Immunology, vol. 147, no. 4, pp. 1153-1162, 1991.

[42] K. Simons and E. Ikonen, "Functional rafts in cell membranes,” Nature, vol. 387, no. 6633, pp. 569-572, 1997.

[43] A. Viola, S. Schroeder, Y. Sakakibara, and A. Lanzavecchia, "T lymphocyte costimulation mediated by reorganization of membrane microdomains," Science, vol. 283, no. 5402, pp. 680-682, 1999.

[44] M. Y. Pu, A. A. Akhand, M. Kato et al., "Mercuric chloride mediates a protein sulfhydryl modification-based pathway of signal transduction for activating Src kinase which is independent of the phosphorylation/dephosphorylation of a carboxyl terminal tyrosine," Journal of Cellular Biochemistry, vol. 63, no. 1, pp. 104-114, 1996.

[45] A. A. Akhand, T. Ikeyama, S. Akazawa et al., "Evidence of both extra- and intracellular cysteine targets of protein modification for activation of RET kinase," Biochemical and Biophysical Research Communications, vol. 292, no. 4, pp. 826$831,2002$.

[46] A. Parashar, A. A. Akhand, R. Rawar et al., "Mercuric chloride induces increases in both cytoplasmic and nuclear free calcium ions through a protein phosphorylation-linked mechanism," Free Radical Biology and Medicine, vol. 26, no. 1-2, pp. 227231, 1999. 
[47] K. Hossain, Y. Kawamoto, M. Hamada et al., "1,4-butanediylbismethanethiosulfonate (BMTS) induces apoptosis through reactive oxygen species-mediated mechanism," Journal of Cellular Biochemistry, vol. 108, no. 5, pp. 1059-1065, 2009.

[48] A. A. Akhand, K. Hossain, H. Mitsui et al., "Glyoxal and methylglyoxal trigger distinct signals for MAP family kinases and caspase activation in human endothelial cells," Free Radical Biology and Medicine, vol. 31, no. 1, pp. 20-30, 2001.

[49] A. A. Akhand, K. Hossain, M. Kato et al., "Glyoxal and methylglyoxal induce lyoxal and methyglyoxal induce aggregation and inactivation of ERK in human endothelial cells," Free Radical Biology \& Medicine, vol. 31, pp. 1228-1235, 2001.

[50] I. Nakashima, W. Liu, A. A. Akhand et al., "4-hydroxynonenal triggers multistep signal transduction cascades for suppression of cellular functions," Molecular Aspects of Medicine, vol. 24, no. 4-5, pp. 231-238, 2003.

[51] D. Barford, A. J. Flint, and N. K. Tonks, "Crystal structure of human protein tyrosine phosphatase 1B," Science, vol. 263, no. 5152, pp. 1397-1404, 1994.

[52] M. D. Resh, "Myristylation and palmitylation of Src family members: the fats of the matter," Cell, vol. 76, no. 3, pp. 411413, 1994.

[53] K. Nakamura, T. Hori, N. Sato, K. Sugie, T. Kawakami, and J. Yodoi, "Redox regulation of a src family protein tyrosine kinase p56(lck) in T cells," Oncogene, vol. 8, no. 11, pp. 31333139, 1993.

[54] M. Kato, T. Iwashita, A. A. Akhand et al., "Molecular mechanism of activation and superactivation of Ret tyrosine kinases by ultraviolet light irradiation," Antioxidants and Redox Signaling, vol. 2, no. 4, pp. 841-849, 2000.

[55] Y. Kawamoto, K. Takeda, Y. Okuno et al., "Identification of RET autophosphorylation sites by mass spectrometry," Journal of Biological Chemistry, vol. 279, no. 14, pp. 14213-14224, 2004.

[56] T. Senga, K. Miyazaki, K. Machida et al., "Clustered cysteine residues in the kinase domain of v-Src: critical role for protein stability, cell transformation and sensitivity to herbimycin A," Oncogene, vol. 19, no. 2, pp. 273-279, 2000.

[57] K. Takeda, Y. Kawamoto, Y. Okuno et al., "A PKC-mediated backup mechanism of the MXXCW motif-linked switch for initiating tyrosine kinase activities," FEBS Letters, vol. 580, no. 3, pp. 839-843, 2006.

[58] M. Kato, K. Takeda, Y. Kawamoto et al., "Repair by Src kinase of function-impaired RET with multiple endocrine neoplasia type $2 \mathrm{~A}$ mutation with substitutions of tyrosines in the COOH-terminal kinase domain for phenylalanine," Cancer Research, vol. 62, no. 8, pp. 2414-2422, 2002.

[59] A. A. Akhand, J. Du, W. Liu et al., "Redox-linked cell surfaceoriented signaling for T-cell death," Antioxidants and Redox Signaling, vol. 4, no. 3, pp. 445-454, 2002.

[60] K. Hossain, A. A. Akhand, Y. Kawamoto et al., "Caspase activation is accelerated by the inhibition of arsenite-induced, membrane rafts-dependent Akt activation," Free Radical Biology and Medicine, vol. 34, no. 5, pp. 598-606, 2003.

[61] M. Ushio-Fukai, "Localizing NADPH oxidase-derived ROS," Science's STKE, vol. 2006, no. 349, p. re8, 2006.

[62] M. J. Morgan, Y. S. Kim, and Z. Liu, "Lipid rafts and oxidative stress-induced cell death," Antioxidants and Redox Signaling, vol. 9, no. 9, pp. 1471-1483, 2007.

[63] M. Medina, A. Del Castillo-Olivares, and I. Núñez De Castro, "Multifunctional plasma membrane redox systems," BioEssays, vol. 19, no. 11, pp. 977-984, 1997. 

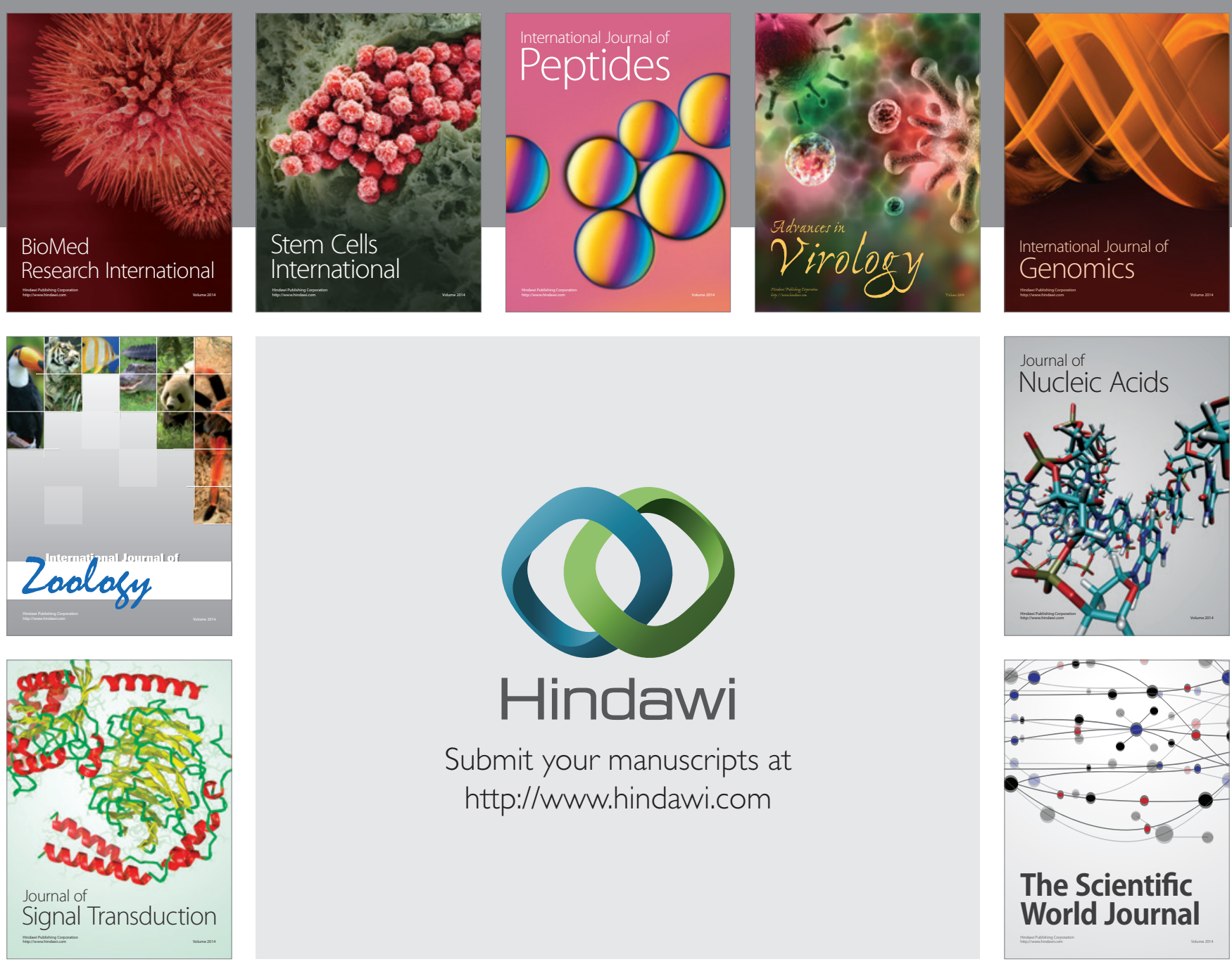

Submit your manuscripts at

http://www.hindawi.com
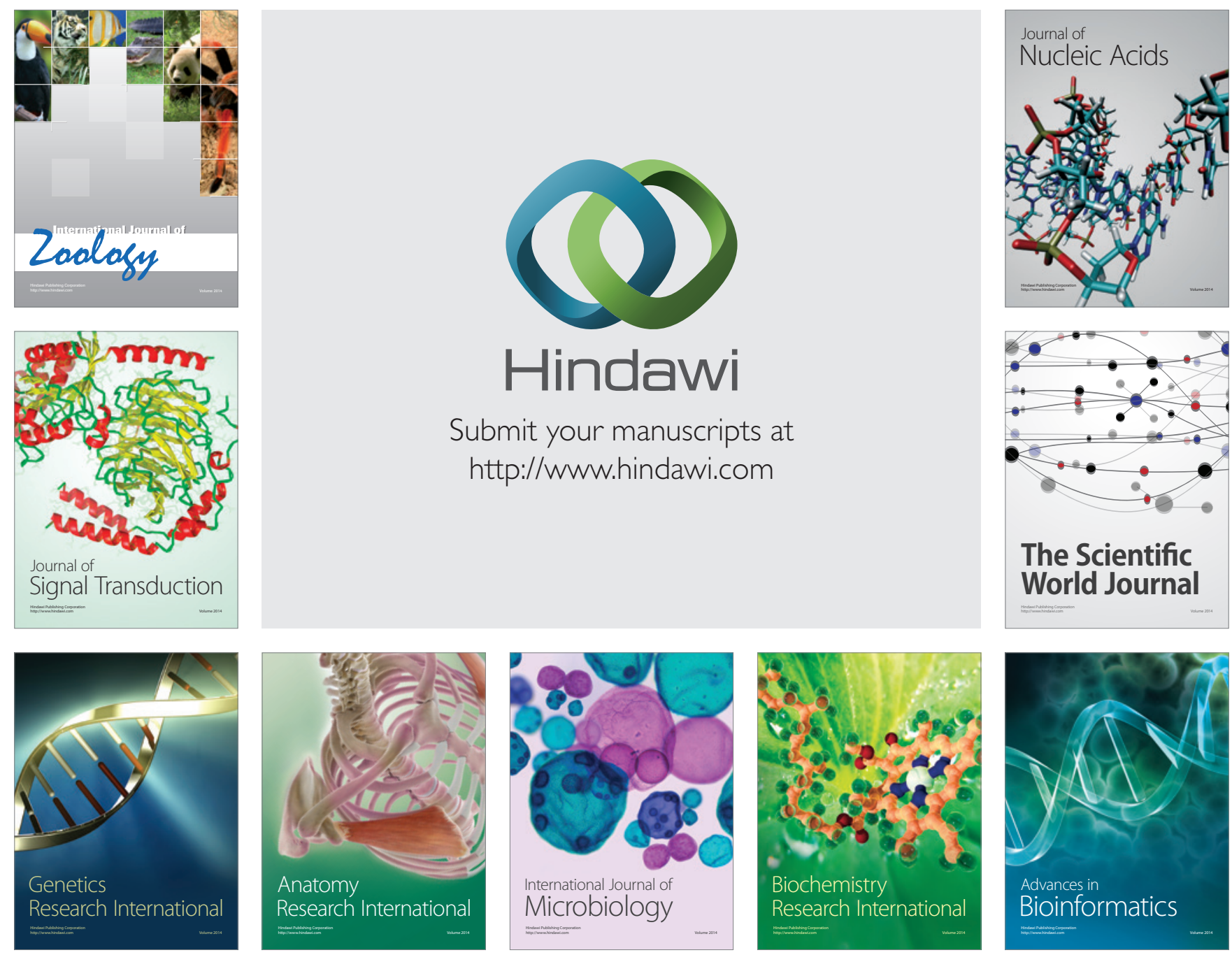

The Scientific World Journal
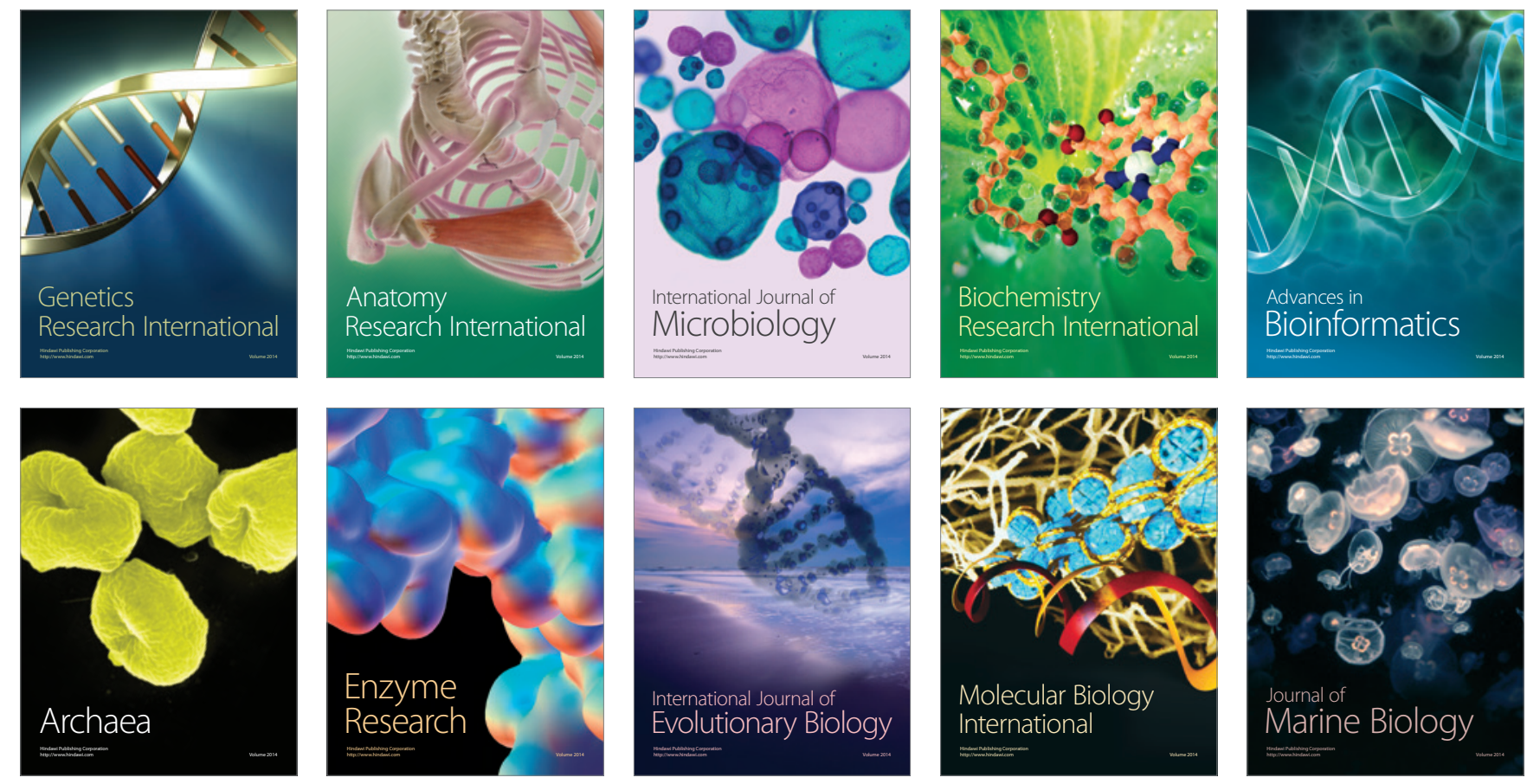